\title{
3D Printed NMR Spectra: From 1D and 2D Acquisition to 3D Visualization
}

Michael Bakker

MSU Graduate Student

Ben Boyd

MSU Graduate Student

Gary A. Meints

Missouri State University

Follow this and additional works at: https://bearworks.missouristate.edu/articles-cnas

Part of the Chemistry Commons

\section{Recommended Citation}

Bakker, Michael, Ben Boyd, and Gary A. Meints. "3D printed NMR spectra: From 1D and 2D acquisition to 3D visualization." Concepts in Magnetic Resonance Part A 47, no. 1 (2018): e21470.

This article or document was made available through BearWorks, the institutional repository of Missouri State University. The work contained in it may be protected by copyright and require permission of the copyright holder for reuse or redistribution.

For more information, please contact BearWorks@library.missouristate.edu. 


\title{
3D printed NMR spectra: From $1 D$ and $2 D$ acquisition to 3D visualization
}

\author{
Michael Bakker | Ben Boyd | Gary A. Meints
}

Department of Chemistry, Missouri State University, Springfield, Missouri

\section{Correspondence}

Gary A. Meints, Department of Chemistry, Missouri State University, Springfield, MO. Email: garymeints@missouristate.edu

\begin{abstract}
Visualization of two-dimensional and relaxation NMR spectra can be difficult for students new to the subject. Educators have utilized 3D printing previously to demonstrate concepts such as potential energy surfaces and molecular orbitals, but this technology has yet to be used to create a visual model of NMR data. In order to address this, we have developed an approach for creating 3D printed models of twodimensional and inversion recovery spectra, which can then be used as an innovative teaching tool. This was done by converting raw FT NMR data into a 3D data graph using Mathematica. This graph is then converted into stereolithography format and printed using a 3D printer. These physical models will allow students to better understand the complex information presented in these NMR spectra.
\end{abstract}

K E Y W O R D S

3D printing, data visualization, NMR relaxation, stereolithography, two-dimensional NMR

\section{1 | INTRODUCTION}

Advanced NMR methods have become more commonplace in the undergraduate chemistry curriculum. ${ }^{1}$ It can be used as a standard technique in organic chemistry classes and inorganic courses as well as physical chemistry courses where both 2D NMR and relaxation are covered. There are also numerous NMR texts aimed at upper-level undergraduates and introductory graduate students to learn NMR in more depth (see reference ${ }^{2}$ for examples). Innovative approaches to teaching 2D NMR have been published recently in the chemistry education literature, ${ }^{3,4}$ in an effort to make a difficult topic more tractable to undergraduates and students new to the concepts. Time-dependent NMR can also readily be incorporated in the undergraduate curriculum. ${ }^{5}$

We developed an approach using 3D printing to visualize NMR data and assist in understanding the utility of 2D NMR for structure determination and $\mathrm{T}_{1}$ data for NMR relaxation. We outline the overall process in a flowchart in Figure 1, and then provide more detail in the Supporting Information. An NMR spectrum is acquired initially, and peak intensities are extracted (either 1D or 2D). These data are then processed by Python (python.org), then converted to a 3D data graph in
Mathematica (Wolfram, Champaign, IL) and finally exported to stereolithography (3D printing,.stl) format for 3D printing. 3D printing technology has become relatively commonplace on university campuses often in conjunction with the library or library services. ${ }^{6}$ With its versatility and tactile nature, 3D printing has a wide variety of applications to chemical education, and provides an alternate way for learners to visualize the relevant information. In particular, some examples where 3D printing has been used for physical chemistry education include 3D printed potential energy surfaces, ${ }^{7-9}$ orbitals, ${ }^{10,11}$ and reaction progress by $3 \mathrm{D}$ visualization of spectroscopic data including 1D NMR. ${ }^{12}$ Additionally, 3D printing can be used to assist students or learners with visual impairments, where the tactile nature can help with conceptualization of the data.

\section{2 | RESULTS AND DISCUSSION}

\section{1 | 2D NMR and 3D printing}


for accreditation by the American Chemical Society. ${ }^{13}$ This highlights the need to offer new and innovative ways for students unfamiliar with NMR to learn the techniques. For the 2D NMR aspect, a ${ }^{1} \mathrm{H}^{-1} \mathrm{H}$ TOCSY (TOtal Correlation SpectroscopY) spectrum has been acquired initially. While COSY experiments offer simpler spectra to analyze, we felt a TOCSY spectrum would be more information-rich and engaging without being overwhelmingly complicated. The sample analyzed is thymidine (Figure 2), the ' $\mathrm{T}$ ' nucleotide in DNA. As with choosing the TOCSY, we felt this molecule offered the right amount of complexity for initial testing of our protocol. The sample was dissolved in one milliliter of 99.9\% deuterium oxide (Cambridge Isotope Labs, Tewksbury, MA). The NMR experiments were performed using a Varian $400 \mathrm{MHz}$ INOVA NMR spectrometer with VnmrJ 4.2 acquisition software. Sample temperature was maintained at $25^{\circ} \mathrm{C}$. The TOCSY experiment used an MLEV17 spinlock of $80 \mathrm{~ms}$, with four scans and $200 \mathrm{t} 1$ increments. The spectral width was set for $4000 \mathrm{~Hz}$ with 1024 complex points, and a presaturation pulse was used for solvent suppression.

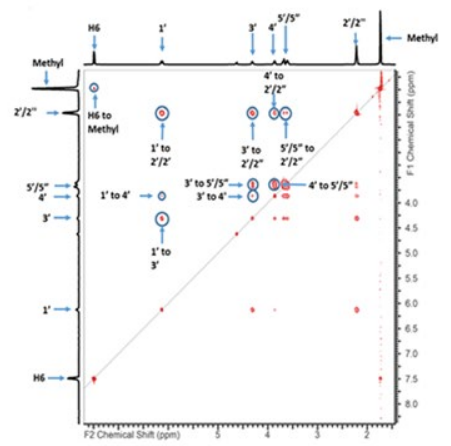

\section{uPrint'se 3D Print Pack}
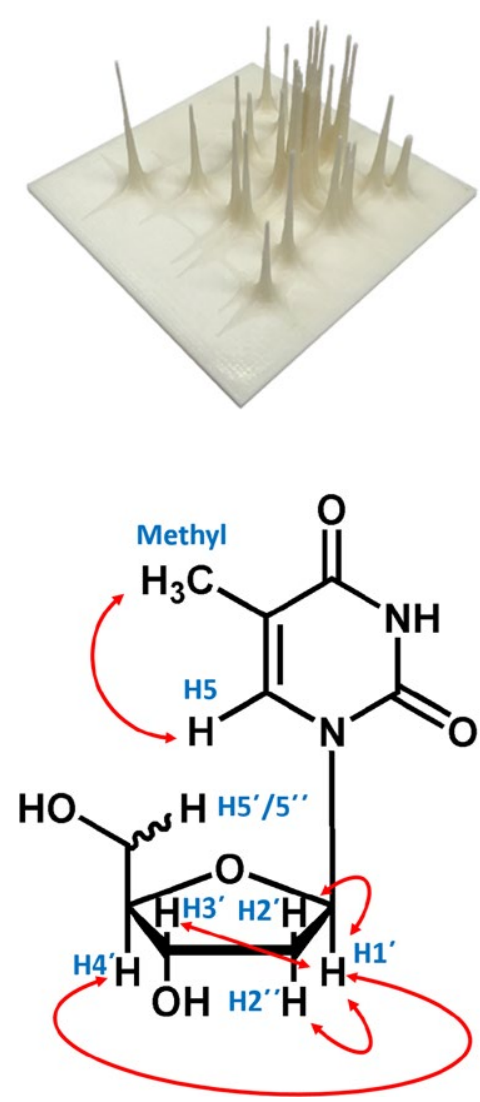
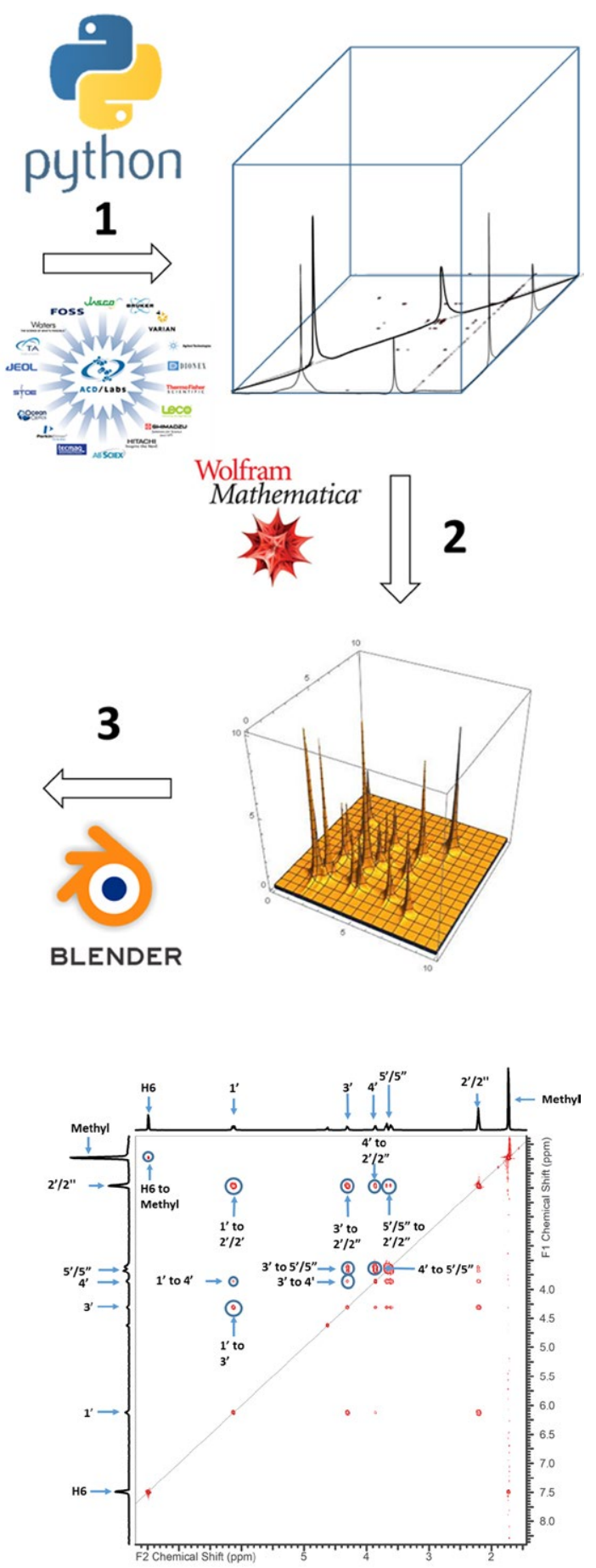

F IG URE 1 Flowchart visualizing how NMR data are converted to a 3D print.

F IG U RE 2 (Left) Thymidine molecule used for the TOCSY experiment. Blue labels indicate the coupled protons. The red arrows indicate several of the connectivites that are shown in the spectrum. (Right) TOCSY spectrum of thymidine as a contour plot, indicating the proton assignments. Note that there are some artefacts associated with the largest (methyl) peak on the diagonal 
After we performed the NMR experiments, they were analyzed using ACD/Spectrus 2017.2.1 software (ACD/Labs, Toronto, Canada). Intensities of the 2D NMR peaks were extracted using the Spectrus Processor. The threshold in Spectrus can be adjusted and the peak picking function can be used to create a list of peaks to export, that represents only the peaks of interest. Alternately, a user could manually pick the peaks of interest. The chosen peaks and intensities are converted into a .txt file through the Export option in the program. The .txt file contains the three values that represent the 2D NMR data: F1, F2, and peak intensity. The specific data used for this work are provided in the Supplemental Information.

From there, a Python program (also provided in the Supporting Information) reads the numbers in the .txt file and creates an output that can be read by Mathematica. As a part of this process, the Python code does the following:

1. Sorts data by intensity and removes all peaks too small to be considered relevant.

2. Normalizes intensity by dividing all intensities by the maximum.

3. Takes the absolute value to create all positive peaks

4. Creates an individual Lorentzian (or Gaussian) function for each of the recorded peaks in $x, y$, and $z$.

5. Generates a mathematical function by converting the matrix into a single string

The result of this process is a command line (step 5, to be read in Mathematica), which is used to generate a 3D graph of the 2D NMR. The spectrum can be adjusted to fit the criteria required for the specified parameters, which in this case were a noise reduction value of 0.05 , a max peak height of five, and a resolution of 100 . These values can be changed to fit an instructor's specific needs. Once completed with the function, the model is symmetrized to fix artefacts by copying missing peaks to the other side of the diagonal and switching the $x$ and $y$ coordinates. Mathematica then allows a user to export a 3D graph as a .stl file, which can then be 3D printed directly. The figure generated by Mathematica can then be visualized in a program such as 3D View (Microsoft Corporation, Redmond,
WA) before 3D printing, to ensure the best quality printed spectrum for the user's needs. The 3D printer used for this paper (in conjunction with the Missouri State Library) is a model uPrint ES, but other models could certainly be used.

To optimize the physical 3D printed model, several iterations and adjustments might need to be performed on the $3 \mathrm{D}$ electronic figure. There likely needs to be a competition between spectral resolution and fragility of the print. If the peaks are thin to show NMR detail (eg, J-couplings) they are prone to breakage. The peaks can be artificially thickened by adjusting the Lorentzian parameters, but NMR information can then be lost. An additional option if losing resolution is not a concern is to use Gaussian functions rather than Lorentzians (Figure 4). While the Lorentzian function is technically more accurate, the Gaussian function is able to provide the same qualitative information, and makes the physical peaks sturdier.

\section{2 $\quad T_{1}$ NMR and 3D printing}

A ${ }^{13} \mathrm{C}$ inversion recovery experiment was performed on 1-hexanol in deuterated methanol, following the work by Gasyna and Jurkiewicz published on the Journal of Chemical Education. ${ }^{7}$ Per standard relaxation analysis, partially relaxed 1D spectra were plotted (Figure 5) as a function of variable time delay $(\tau)$. The intensities are fit with an exponential curve as a function of recovery time and peak intensity, and the $T_{1}$ values extracted. The goal is to use information from these spectra to create a 3D graphical format to visualize in Mathematica, and then 3D printed.

To create a 3D time-dependent NMR model (details in the Supporting Information), we decided to simplify the process as much as possible. The only parameters needed for this are the equilibrium peak intensity $\left(M_{0}\right.$, obtained from a $1 \mathrm{D}$ or fully relaxed spectrum) and the $T_{1}$ values for the peaks that are to be displayed in the 3D print. The 3D figure (Figure 5) is then generated using Equation 1 below:

$$
M_{Z}=M_{0}\left(1-2 e^{\tau / T_{1}}\right)
$$

F I G URE 3 Comparison of the 3D Viewer visualized stacked plot of the TOCSY spectrum and the 3D printed spectrum. Arrow indicates the direction of the diagonal.
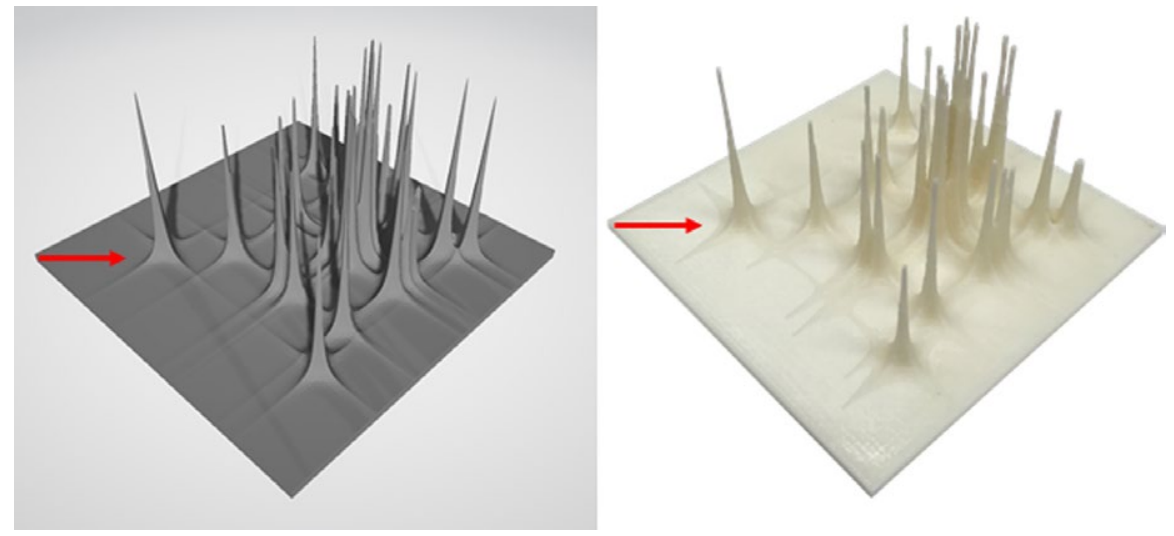

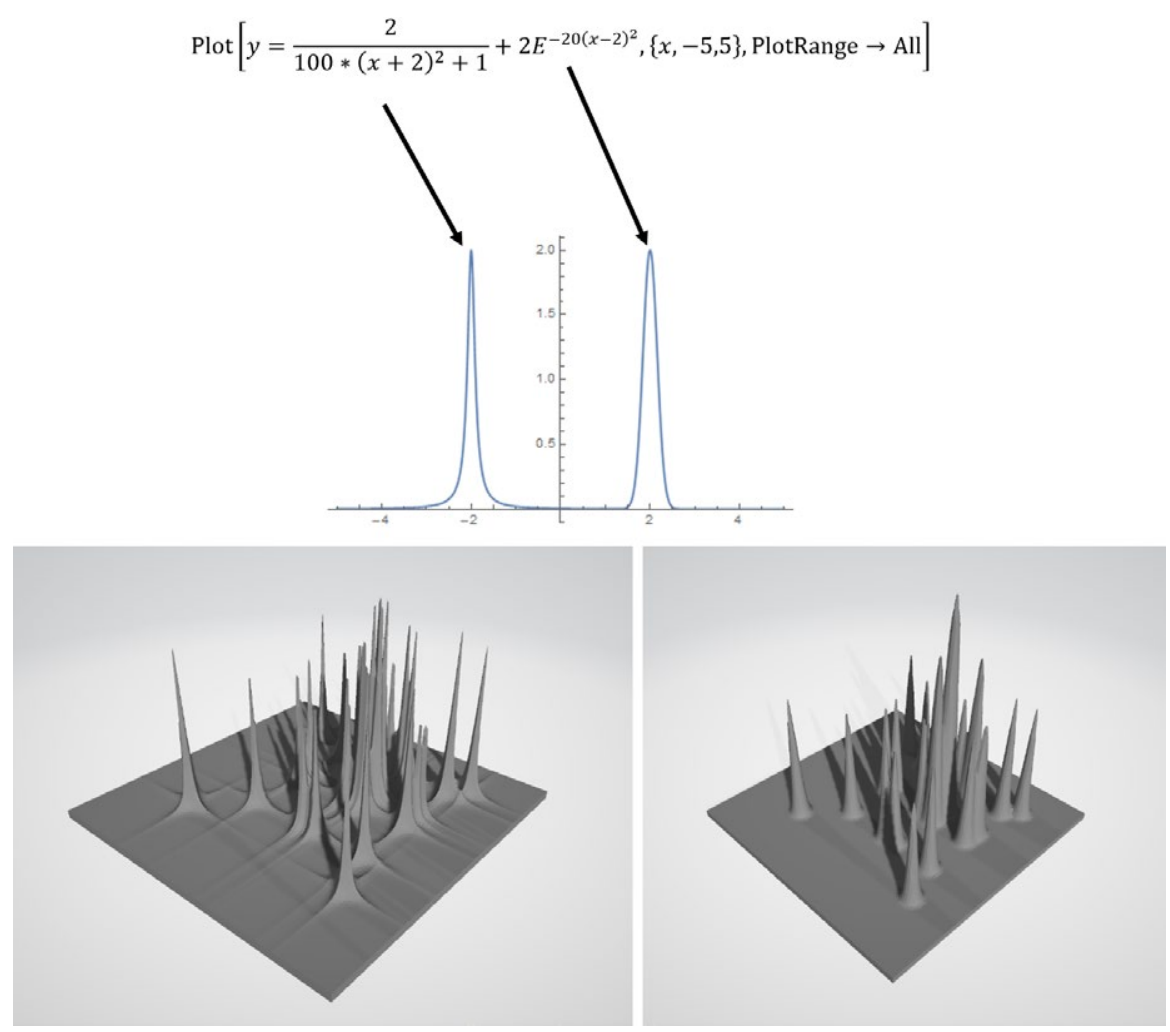

F I G URE 4 (Top) Forms of the Lorentzian and Gaussian equations used to generate the $3 \mathrm{D}$ spectra, and a $2 \mathrm{D}$ projection of how the peaks appear. (Bottom) Comparison of how the different formulations affect the appearance of the 3D figure.
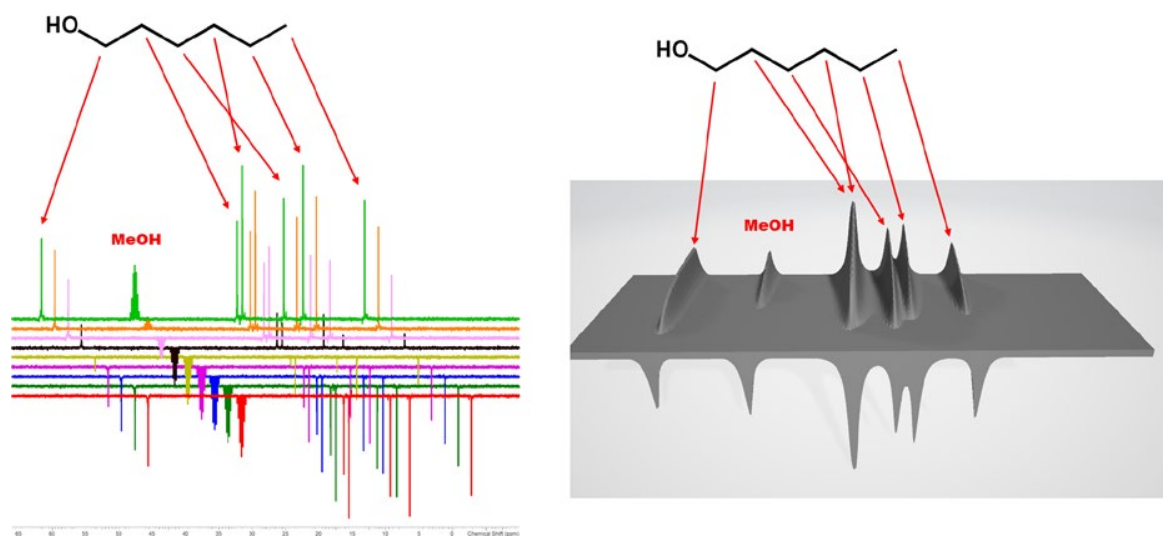

F I G URE 5 (Left) ${ }^{13} \mathrm{C}$ Inversion recovery spectra for 1-hexanol in deuterated methanol. The carbons are specified according to the assignments in reference 5. (Right) Indication of the carbon peaks for the 3D graph of the inversion recovery experiment. The graph and its 3D print are intended to represent the spectra as shown but note that the carbons 2 and 4 have lost resolution. Note that the residual methanol solvent peak is included (at $49.15 \mathrm{ppm}$ ).

\section{3 | CONCLUSION}

Figure 2 shows the 2D TOCSY spectrum as it was acquired and processed. Figure 3 shows the 3D Viewer version next to the 3D printed version of the same spectrum. As can be seen, the relevant crosspeaks that appear in the 2D plane of the NMR spectrum are now able to be visualized as their 3D equivalent. Likewise, Figure 5 shows the inversion recovery experiment and Figure 6 shows a comparison of 3D View to the 3D printed model.

The 3D models generated by this work demonstrate how new technology can be used for innovative teaching options, even on advanced topics such as $2 \mathrm{D}$ and relaxation NMR. Depending on the type of NMR experiment, the entire process could be completed within a day or so. A TOCSY or inversion recovery experiment on a relatively concentrated small molecule can take about 15-20 minutes. The data processing and computations in Python and Mathematica take about 5-20 minutes depending on computer speed. The 3D printing process can be (in our experience) between 2 and 12 hours, and prints were generated overnight without the need for supervision of the printing process. As an example, the Lorentzian 2D print took 4.75 hours, cost $\$ 24$, used 3.45 

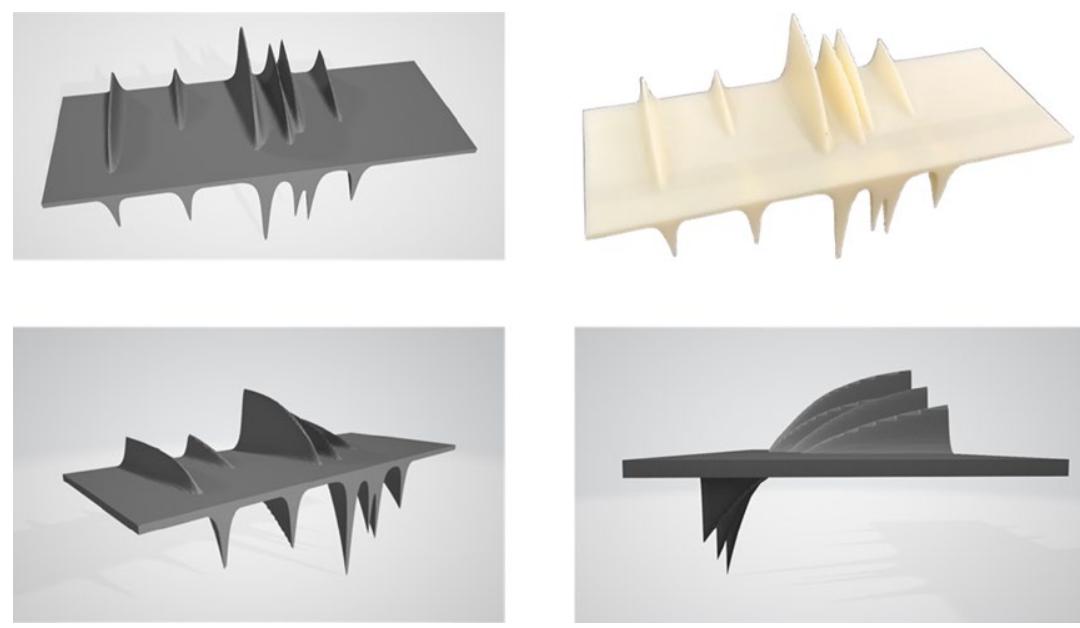

F I G URE 6 (Top) Comparison of 3D visualized inversion recovery and $3 \mathrm{D}$ printed version. (Bottom) Different orientations of the 3D figure, to display the exponential recovery of the magnetization.

\section{REFERENCES}

1. Rovnyak D, Stockland Jr. R (ed). Modern NMR Spectroscopy in Education. ACS Symposium Series 969, Washington, DC: American Chemical Society; 2007.

2. (a) Becker ED. High Resolution NMR: Theory and Applications, 3rd ed. San Diego: Academic Press, 2000. (b) Claridge TDW. High-Resolution NMR Techniques in Organic Chemistry. Amsterdam: Pergamon, 1999. (c) Günther H. NMR Spectroscopy. Chichester, UK: Wiley \& Sons, 1995. (d) Field LD, Li HL, Magill AM. Organic Structures from 2D NMR Spectra. Chichester, UK: Wiley \& Sons, 2016. (e) Simpson JH. Organic Structure Determination Using 2-D NMR Spectroscopy: A Problem-Based Approach. Amsterdam: Elsevier, 2008. (f) Richards SA, Hollerton JC. Essential Practical NMR for Organic Chemistry. Chichester, UK: Wiley \& Sons; 2011.

3. Anderson-Wile AM. Introducing 2D NMR spectroscopy to second-year undergraduate chemistry majors using a building-up approach. J Chem Ed. 2016;93:699-703.

4. Graham KJ, McIntee EJ, Schaller CP. Web-based 2D NMR spectroscopy practice problems. J Chem Ed. 2016;93:1483-1485.

5. Gasyna ZL, Jurkiewicz AJ. Determination of Spin-lattice relaxation time using 13C NMR: an undergraduate physical chemistry laboratory experiment. J Chem Educ. 2004;81:1038-1039.

6. Bharti N, Singh S. Three-dimensional (3D) printers in libraries: perspective and preliminary safety analysis. J Chem Educ. 2017;94:879-885.

7. Kaliakin DS, Zaari RR, Varganov SA. 3D printed potential and free energy surfaces for teaching fundamental concepts in physical chemistry. J Chem Educ. 2015;92:2106-2112.

8. Lolur P, Dawes R. 3D printing of molecular potential energy surface models. J Chem Educ. 2014;91:1181-1184.

9. Teplukhin A, Babikov D. Visualization of potential energy function using an isoenergy approach and $3 \mathrm{D}$ prototyping. J Chem Educ. 2015;92:305-309.

10. Griffith KM, de Cataldo R, Fogarty KH. Do-it-yourself: 3D models of hydrogenic orbitals through $3 \mathrm{D}$ printing. J Chem Educ. 2016;93:1586-1590.

11. Robertson MJ, Jorgensen WL. Illustrating concepts in physical organic chemistry with $3 \mathrm{D}$ printed orbitals. J Chem Educ. 2015;92:2113-2116. 
12. Higman CS, Situ H, Blacklin P, Hein JE. Hands-on data analysis: using 3D printing to visualize reaction progress surfaces. J Chem Educ. 2017;94:1367-1371.

13. ACS Guidelines and Evaluation Procedures for Bachelor's Degree Programs. https://www.acs.org/content/dam/acsorg/about/governance/committees/training/2015-acs-guidelines-for-bachelors-degree-programs.pdf. Accessed November 26, 2018.

14. What is 2D NMR? https://www.theresonance.com/what-is-2dnmr/. Accessed November 28, 2018.

\section{SUPPORTING INFORMATION}

Additional supporting information may be found online in the Supporting Information section at the end of the article.
The .stl and Python files are available at https://bearworks. missouristate.edu/articles-cnas/25/.

How to cite this article: Bakker M, Boyd B, Meints GA. 3D printed NMR spectra: From 1D and 2D acquisition to 3D visualization. Concepts Magn Reson Part A. 2019;47A:e21470. https://doi.org/10.1002/ cmr.a.21470 